\title{
Tratamentos superficiais de sistemas cerâmicos para união a cimentos resinosos
}

\section{Surface treatments of ceramic systems for bonding to resin cements}

\author{
Verônica Pereira de Lima*
}

\section{Resumo}

Objetivo: apresentar uma revisão de literatura sobre os tipos de tratamentos de superfície disponíveis para cimentação adesiva a sistemas cerâmicos. Revisão de literatura: ainda que haja certo consenso na união adesiva às vitrocerâmicas, ainda não há uma unanimidade quanto à adesão de cerâmicas policristalinas de alta resistência. Com base na literatura disponível, para vitrocerâmicas, o tratamento preferencial ainda é o condicionamento com ácido hidrofluorídrico (HF), em concentração e tempo correspondentes a seu conteúdo cristalino, seguido de silanização. Para as policristalinas à base de zircônia, métodos como condicionamento seletivo por infiltração ou revestimento triboquímico com sílica, seguidos de silanização e utilização de primers ou cimentos resinosos contendo monômero MDP, resultaram em altos valores de resistência de união. Considerações finais: vitrocerâmicas são adequadamente tratadas por meio de condicionamento com HF, seguido de silanização. Maiores valores de resistência de união para as cerâmicas policristalinas foram obtidos após tratamento com condicionamento seletivo por infiltração ou silicatização, seguido de silanização, além da utilização de primers ou cimentos resinosos contendo monômero MDP. Porém, são necessários estudos in vitro adicionais para definir a melhor opção de tratamento para tais materiais.

Palavras-chave: Cerâmicas. Cimentação. Restauração dentária permanente.

\section{Introdução}

A maior exigência por materiais restauradores com excelentes qualidades estéticas e estabilidade de cor em longo prazo expandiu o emprego de sistemas cerâmicos em tratamentos reabilitadores ${ }^{1}$. Considerando a natureza friável das cerâmicas, buscou-se melhorar as propriedades mecânicas de tais materiais por meio do desenvolvimento de novas formas de processamento e do aumento do conteúdo cristalino ${ }^{2-4}$. Como resultado de tais melhorias, ampliaram-se as opções de materiais cerâmicos disponíveis para uso odontológico bem como suas indicações. Considerando sua composição, esses materiais podem ser agrupados em três classes principais: vitrocerâmicas, cerâmicas vítreas com partículas de carga ou parcialmente cristalinas e cerâmicas cristalinas ou policristalinas ${ }^{5}$.

Tais avanços, em associação com o desenvolvimento dos agentes adesivos, ampliaram a indicação de restaurações totalmente cerâmicas minimamente invasivas, por não dependerem exclusivamente da retenção mecânica, com obtenção de excelentes resultados estéticos ${ }^{6}$. Os procedimentos adesivos são fundamentais para o sucesso das restaurações cerâmicas, uma vez que falhas na adesão podem resultar em descolamento ou fratura do material ${ }^{7,8}$. Além disso, uma união efetiva provê retenção e melhora a adaptação marginal da restauração ${ }^{9}$.

Mestre em Ciências Odontológicas, Universidade Federal do Amazonas. Especialista em Prótese Dentária, Uninorte. Doutoranda em Materiais Dentários, Programa de Pós-Graduação em Odontologia da Faculdade de Odontologia, Universidade Federal de Pelotas, Rio Grande do Sul, Brasil. 
Assim, conforme o sistema cerâmico escolhido, o tipo de cimento e o procedimento de cimentação devem ser selecionados de forma adequada para proporcionar maior resistência de união à interface cerâmica $/$ resina $^{10}$. Se a opção for uma vitrocerâmica, por se tratar de material empregado há mais tempo, o procedimento é mais previsível, com resultados duradouros quando os protocolos de condicionamento e cimentação da peça são seguidos ${ }^{11}$. Porém, materiais recentes que apresentam maior resistência, como as cerâmicas policristalinas, diferem das vitrocerâmicas quanto a composição e propriedades físicas, requerendo técnicas adesivas alternativas para atingir uma união estável ao cimento resinoso ${ }^{12}$.

A fim de melhorar a resistência de união do cimento à superfície cerâmica, são propostas várias estratégias para o tratamento superficial das cerâmicas odontológicas, desde condicionamento com ácido hidrofluorídrico (HF), seguido de aplicação de silano ${ }^{8,13-15}$, até jateamento com óxido de alumínio ${ }^{16-18}$, revestimento com partículas modificadas por sílica ${ }^{18,19}$ e, mais recentemente, emprego de diferentes tipos de laser ${ }^{20-22}$. É importante considerar as características dos cimentos resinosos e sistemas adesivos para que, combinados ao tratamento de superfície, possam proporcionar resultados de resistência adesiva melhorados ${ }^{23,24}$. Assim, esta revisão de literatura tem o objetivo de avaliar estudos in vitro sobre o efeito de tratamentos de superfície na resistência de união de cimentos resinosos às cerâmicas odontológicas.

\section{Materiais e método}

A revisão de literatura utilizou artigos completos publicados na base de dados eletrônica PubMed. A busca na literatura foi realizada no mês de dezembro de 2017, combinando descritores relacionados a: ceramic, surface treatment, bond strength $\mathrm{e}$ resin cement.

Os artigos foram selecionados com base em leitura inicial do título e do resumo de publicações dos últimos dez anos (a partir de 2007). Após a triagem inicial, foi feita a leitura na íntegra dos documentos relevantes que envolvessem a aplicação de tratamentos superficiais em cerâmicas odontológicas. Foram incluídos artigos publicados em língua inglesa de pesquisas originais que testaram aplicação de tratamentos de superfície em sistemas cerâmicos. Foram excluídos manuscritos em outros idiomas, artigos não disponíveis em texto completo e publicações não condizentes à temática proposta. Os estudos elegíveis também tiveram as suas referências consultadas.

\section{Revisão de literatura}

\section{Vitrocerâmicas}

As vitrocerâmicas apresentam características altamente estéticas, capazes de mimetizar as propriedades ópticas do esmalte e da dentina, empregadas em restaurações totalmente cerâmicas desde os anos $1970^{5}$. Os vidros em cerâmicas odontológicas são provenientes principalmente de feldspato e são constituídos à base de sílica (óxido de silício) e alumina (óxido de alumínio). As vitrocerâmicas apresentam uma rede de átomos dispostos de forma tridimensional, sem um padrão regular de distância e ângulo entre si, tornando essa rede menos densa, irregular e, consequentemente, menos resistente à propagação de trincas ${ }^{11}$. Durante o aquecimento, o feldspato pode formar cristais de leucita, cuja presença é uma maneira de controlar a expansão térmica da vitrocerâmica e possibilita o uso de tais cerâmicas como recobrimento de coroas com infraestruturas de metal ${ }^{7}$.

Apesar de sua natureza frágil, é possível aumentar a resistência de restaurações vitrocerâmicas por meio da cimentação adesiva com cimento resinoso ${ }^{25}$. Tais cerâmicas são também classificadas como ácido-sensíveis (cerâmicas à base de feldspato, leucita e dissilicato de lítio), pois, quando condicionadas com ácido hidrofluorídrico (HF), ocorre a dissolução dos componentes vítreos superficiais e a produção de uma superfície mais porosa e rugosa, que facilita a penetração do agente cimentante nas microrretenções ${ }^{2,13}$. Tal condicionamento, juntamente com a aplicação de um agente de união bifuncional (silano), forma o padrão para obtenção de adesão a essas cerâmicas ${ }^{26,27}$.

\section{Cerâmicas vítreas com partículas de carga ou parcialmente cristalinas}

As cerâmicas vítreas com partículas de carga ou parcialmente cristalinas podem apresentar conteúdo vítreo variado, exibindo alto ou baixo teor de vidro em sua composição. A adição de conteúdo cristalino a este grupo visa melhorar as propriedades mecânicas das cerâmicas vítreas ${ }^{7}$. Essa categoria abrange cerâmicas com diversas proporções entre matriz vítrea e partículas cristalinas, além de diferentes tipos de cristais ${ }^{7,26,28,29}$.

A leucita foi o primeiro tipo de cristal adicionado às vitrocerâmicas, com o objetivo de aumentar seu coeficiente de expansão térmico ${ }^{7}$. Em geral, $17 \%$ a $25 \%$ de leucita são adicionados às cerâmicas vítreas para torná-las mais compatíveis às ligas metálicas durante a queima ${ }^{7,26}$, variando conforme o sistema cerâmico. Em maiores concentrações, de $35 \%$ a $55 \%$, a leucita é utilizada como reforço, pois os cristais atuam com barreira para a propagação de trincas ${ }^{7,26}$. Outra possibilidade de adição é a de 
cristais de dissilicato de lítio, porém em concentrações bem maiores, $70 \%$ de volume, aumentando sua resistência final ${ }^{26}$. As cerâmicas vítreas reforçadas com leucita e dissilicato de lítio, por ainda possuírem grande quantidade de matriz vítrea, podem ser condicionadas por HF, sendo consideradas ácido-sensíveis ${ }^{13,26}$.

Ainda nesse grupo de cerâmicas parcialmente cristalinas, outros sistemas podem ser infiltrados por vidro, contendo de $70 \%$ a $80 \%$ em peso de óxido de alumínio, o que resulta em alta resistência ${ }^{26,28}$. Outros metais, como óxido de zircônio ou o espinélio aluminato de magnésio, podem ser utilizados como modificação do sistema infiltrado por vidro original, buscando otimizar sua resistência, no caso do óxido de zircônio combinado à matriz de alumina, ou aumentar a translucidez, no caso do espinélio ${ }^{12,20,30}$. Todavia, tal ganho em propriedades mecânicas ou ópticas pela incorporação cristalina a essas cerâmicas alterou seu padrão de reatividade química aos ácidos.

Em geral, quanto maior o conteúdo cristalino em um sistema cerâmico, menos reativo ele se torna aos ácidos, resultando em cerâmicas ácido-resistentes que mostram pouca degradação superficial pelo condicionamento com HF, impedindo a obtenção de uma união micromecânica confiável ${ }^{31}$. Tal reatividade também é influenciada pela orientação e pelos tipos dos cristais. Como no caso dessas cerâmicas o condicionamento com HF não é suficiente para promover união adequada, são necessárias outras abordagens para tratar a superfície antes da adesão, como o revestimento triboquímico por sílica ${ }^{19,32}$.

\section{Cerâmicas cristalinas ou policristalinas}

O grupo das cerâmicas cristalinas ou policristalinas não apresenta fase vítrea, mas uma matriz atômica regular densa que dificulta a propagação de trincas ${ }^{18}$. Por esse motivo, cerâmicas policristalinas são, geralmente, mais resistentes e menos estéticas do que as cerâmicas vítreas, porém, seu processamento é mais complexo, sendo realizado pelo sistema CAD/CAM (Computer-aided design/ Computer-aided manufacturing). Existem várias opções de sistemas cerâmicos formados pela sinterização direta de cristais disponíveis comercialmente, tanto os de alumina (Procera AllCeram, Nobel Biocare) quanto os à base de zircônia (Cercon, Dentsply; LAVA, 3M ESPE; Procera AllZirkon, Nobel Bio(are $)^{4,18}$.

Em geral, as cerâmicas com alto teor cristalino são mais opacas, se comparadas com as vitrocerâmicas, assim, são indicadas principalmente como material de infraestrutura sobre o qual uma vitrocerâmica de cobertura é aplicada, para alcançar melhores resultados estéticos ${ }^{4,18}$. As cerâmicas policristalinas também são consideradas ácido-resistentes, pois não apresentam fase vítrea e sua superfície não pode ser condicionada por $\mathrm{HF}^{31}$.

\section{Tratamento de superfície}

O êxito da adesão entre estrutura dentária e restauração cerâmica depende das propriedades adesivas e da resistência de união do cimento resinoso. O que a maioria dos tratamentos tem em comum é a modificação da superfície cerâmica visando aumentar a retenção micromecânica bem como a união química ${ }^{11}$.

Em cerâmicas ácido-sensíveis, o HF promove uma dissolução superficial por atacar seletivamente a fase vítrea dessas cerâmicas, expondo o dióxido de silício $\left(\mathrm{SiO}_{2}\right)$ e produzindo alterações topográficas que contribuem para retenção micromecânica e união química, quando o silano é empregado ${ }^{25}$. O condicionamento ácido juntamente com a silanização promovem molhabilidade ao cimento na superfície cerâmica, além de alterar sua energia superficial e, consequentemente, seu potencial adesivo à resina $^{31}$.

Em um estudo ${ }^{31}$ que comparou as mudanças nos padrões estruturais de superfície de uma cerâmica feldspática de acordo com a concentração do HF $(1 \%$, $3 \%, 5 \%$ ou $10 \%$ ), mesmo a mínima modificação superficial gerada pelo condicionamento resultou em redução na resistência flexural, sem diferença entre as concentrações testadas. Além disso, o efeito do condicionamento na rugosidade foi progressivo, ou seja, quanto maior a concentração de $\mathrm{HF}$, maior a irregularidade produzida na superfície da cerâmica.

Contudo, ainda que o protocolo de cimentação adesiva à porcelana feldspática seja bem estabelecido na literatura, há alguma controvérsia quanto à ótima concentração de HF necessária para promover resistência de união durável, sem enfraquecer as cerâmicas. Em outro estudo ${ }^{25}$ comparando diferentes concentrações de $\mathrm{HF}(1 \%, 3 \%, 5 \%$ e $10 \%)$, observou-se que o condicionamento reduziu significativamente os valores de ângulo de contato, o que resultou em aumento de molhabilidade. Houve, ainda, produção de mudanças no padrão topográfico para todas as concentrações, porém apenas as concentrações de $3 \%, 5 \%$ e $10 \%$ promoveram valores de resistência de união estáveis e similares, enquanto HF $1 \%$ gerou resistência de união instável.

Há, na literatura, estudos que mostram efetividade de concentrações de HF para condicionamento de cerâmicas nas concentrações de $2,5 \%$ a $10 \%$, sendo aplicadas por diferentes intervalos de tempo, variando de 60 segundos a 3 minutos $^{25,29,33}$. No entanto, é necessário considerar o tempo ótimo de condicionamento conforme o conteúdo cristalino de uma determinada cerâmica, pois o sobrecondicionamento pode reduzir a resistência de união, ao invés de aumentá-la. Em porcelanas com baixo a moderado conteúdo de leucita ( $17 \%$ a $25 \%)$, os maiores valores de resistência de união foram obtidos no condicionamento com gel de $\mathrm{HF}$ a $9,5 \%$ por 60 segun$\operatorname{dos}^{26}$. Já, para aquelas com alto conteúdo de leucita (35\% a 55\%), o tempo para atingir uma resistência 
de união adequada foi cerca de três vezes maior, de 150 a 180 segundos $^{26}$.

Em cerâmicas vítreas à base de dissilicato de lítio, cujo conteúdo de carga é aproximadamente de $70 \%$, o condicionamento com $\mathrm{HF}$ a $5 \%$ por 20 segundos é considerado padrão para obtenção de adesão adequada ${ }^{26,27,34}$. Porém, para melhorar tal união, pode-se combinar a esse tratamento convencional uma abrasão com partículas a ar antes do condicionamento ácido com HF e silanizacão. A abrasão e o condicionamento dissolvem seletivamente a fase vítrea e expõem os cristais de dissilicato de lítio, servindo de recursos para aumentar a retenção ${ }^{29}$.

Ainda que o condicionamento com HF exerça importante papel na adesão de cerâmicas ao cimento resinoso, tais alterações superficiais não são suficientes se não forem seguidas de adequada silanização. Em um estudo que avaliou a resistência de união à microtração entre cimento resinoso e cerâmica feldspática submetida a diferentes regimes de condicionamento, com e sem aplicação de silano, observou-se maiores valores de resistência de união para os grupos tratados com silano, independente do condicionamento. Tais valores permaneceram estáveis mesmo após teste de envelhecimento por termociclagem e 150 dias de armazenamento em água $^{13}$. Tal efeito é observado devido à característica bifuncional da molécula de silano, que se une ao dióxido de silício presente na superfície cerâmica por meio dos radicais hidroxila, enquanto que, na outra extremidade, o grupamento funcional interage com a matriz orgânica da resina.

Como já apresentado, algumas cerâmicas possuem pouco conteúdo vítreo ou nenhuma fase vítrea. No caso de cerâmicas vítreas parcialmente cristalinas à base de óxido de alumínio, como no sistema In-Ceram Alumina, o condicionamento ácido não promove suficiente alteração superficial de modo a promover a adesão ${ }^{28}$. O jateamento com partículas de $250 \mu \mathrm{m}$ de alumina é um método efetivo para criar o efeito equivalente ao do condicionamento com HF em vitrocerâmicas, pois gera uma superfície ativada e asperizada, seguido de silanização ${ }^{35}$. No entanto, maiores valores de resistência de união foram observados quando empregado jateamento de partículas de alumina de $110 \mu \mathrm{m}$ revestidas por sílica, sob pressão (Sistema Rocatec Plus, 3M ESPE, St. Paul, MN, EUA), seguido de silanização ${ }^{26,27}$. A vantagem desse condicionamento, também chamado revestimento triboquímico por sílica ou silicatização, é que as partículas revestidas de sílica são incorporadas na superfície do substrato, deixando-a parcialmente coberta com sílica. A sílica então exposta na camada superficial possibilita que o agente silano forme uma união durável com o substrato ${ }^{19,32}$.

Os sistemas cerâmicos policristalinos, por serem ácido-resistentes ${ }^{19,32}$, constituem um desafio em termos de tratamento superficial visando a uma adesão ótima. Além dos tratamentos já citados, outros métodos vêm sendo testados para tratamento de superfície de cerâmicas sem fase vítrea. Um método é o condicionamento seletivo por infiltração, no qual a superfície de zircônia é revestida com uma fina camada de agente condicionador contendo vidro. Este agente é aquecido até que ocorra transição, e o vidro fundido permite o deslizamento dos grãos na superfície, aumentando a tensão superficial. Em seguida, o vidro é lavado em um banho de ácido, para voltar à temperatura ambiente. Esse procedimento gera uma superfície retentiva, melhorando a estabilidade hidrolítica da união. Alguns estudos mostraram que tal método, combinado à silanização, melhorou a ligação da zircônia à resina ${ }^{36,37}$.

O laser de Er:YAG (érbio: ítrio - alumínio - granada) atua por remover as partículas da superfície por meio de microexplosões e ablação por vaporização. Tais efeitos podem ser acompanhados de mudanças de temperatura, que podem ser prejudiciais, possivelmente, induzindo à transformação de fases. Ainda que promissores, o tipo efetivo de laser e seu modo de aplicação ainda não estão claramente definidos na literatura, sendo ainda necessárias mais investigações ${ }^{38,39}$.

Ainda assim, resultados positivos no emprego de laser como tratamento superficial de cerâmi$\operatorname{cas}^{38,40}$ já foram relatados. Akin et al. ${ }^{40}$ (2012) observaram maiores valores de resistência de união para as cerâmicas à base de zircônia tetragonal estabilizada por ítrio (Y-TZP), tratadas com laser Er:YAG. Em outro estudo ${ }^{38}$, tanto o laser de $\mathrm{CO}_{2}$ (dióxido de carbono) quanto o laser de Er:YAG foram testados e se mostraram efetivos em promover adequada resistência de união ao cisalhamento entre zircônia e cimento resinoso.

Considerando que uma união efetiva não depende somente das alterações de superfície produzidas pelos diversos tratamentos de superfície, mas também da capacidade de adesão química à superfície, o emprego do monômero 10-metacriloxidecil dihidrogênio fosfato (10-MDP) em agentes adesivos resinosos tem se mostrado uma opção interessante na cimentação de cerâmicas à base de zircônia ${ }^{24}$. Originalmente, foi desenvolvido para união a óxidos metálicos, mas, devido à característica da zircônia de exibir uma camada superficial de óxido passivo, acaba se comportando similarmente aos metais ${ }^{23}$. Observou-se, em alguns estudos, um aumento na resistência de união à zircônia quando são usados primers ou cimentos resinosos contendo o monômero MDP após o jateamento a ar ${ }^{41,42}$. Em um estudo in vitro que avaliou o efeito de diferentes tratamentos superficiais e do monômero MDP na resistência de união ao cisalhamento entre zircônia e cimento resinoso, observou-se que o uso de cimento resinoso contendo MDP aumenta a resistência de união da zircônia jateada ${ }^{23}$. Apesar de promover melhora na resistência de união ao empregar materiais contendo MDP, ainda se faz necessário obter retenção mecânica adicional para adequada adesão à zircônia ${ }^{43}$. 


\section{Discussão}

A qualidade da união adesiva entre cimentos resinosos e restaurações cerâmicas é significativamente influenciada pelo tratamento da superfície empregado, promovendo união química e micromecânica ao tecido dentário ${ }^{9,10}$. A melhoria gradual dos sistemas cerâmicos levou ao desenvolvimento de materiais com diferentes propriedades mecânicas e características ópticas, com grande diversidade na composição dos sistemas, abrangendo desde sistemas predominantemente vítreos até cerâmicas totalmente cristalinas ${ }^{1,5,6,28}$. Em contrapartida, tal variedade estrutural e de superfície entre os materiais cerâmicos resultou em diferentes padrões de condicionamento, conforme o agente condicionante utilizado, o tempo de aplicação e a concentração.

As vitrocerâmicas, por sua matriz vítrea predominante, são adequadamente condicionadas com $o$ $\mathrm{HF}^{2,3,11}$. O condicionamento com HF promove uma porosidade uniforme na superfície de vitrocerâmicas, de sistemas à base de dissilicato de lítio e de leucita ${ }^{7,11,13,29}$. Ainda que sejam ácido-sensíveis, tais cerâmicas apresentam diferenças estruturais e de composição, logo, a concentração do ácido e o tempo de condicionamento ideal variam entre elas. Para as vitrocerâmicas, há na literatura evidências de um padrão adequado de condicionamento obtido com HF em concentrações de $2,5 \%$ a $10 \%$, aplicadas por períodos de 60 segundos a 3 minutos ${ }^{25,29,33}$. Para cerâmicas vítreas com baixo conteúdo de leucita, o condicionamento com HF a 9,5\% por 60 segundos resultou em adequados valores de resistência de uniã $0^{5,26}$. Já, para cerâmicas vítreas à base de dissilicato de lítio, HF a $5 \%$ durante 20 segundos é suficiente para obtenção de uma adesão adequada ${ }^{26,27,34}$. Sendo assim, mesmo entre sistemas cerâmicos ácido-sensíveis, é importante levar em consideração informações sobre a composição, frequentemente fornecidas pelo fabricante, a fim de produzir apenas o grau de condicionamento necessário e não gerar sobre ou subtratamento da restauração cerâmica utilizada.

Nas cerâmicas parcialmente cristalinas e nas policristalinas, foram obtidos bons resultados na união quando realizado o jateamento com partículas de alumina ou com partículas de alumina revestidas por sílica (silicatização) ${ }^{26-28}$. Com o jateamento com partículas de alumina, ocorre a criação de irregularidades superficiais ou de rugosidade, o que melhora a retenção micromecânica da superfície, de maneira semelhante ao padrão de irregularidade produzido pelo condicionamento com HF. Já, na silicatização, além das irregularidades geradas pelo jateamento que aumentam a microrretenção, como essas partículas de alumina estão modificadas por sílica, há um aumento também na quantidade de sílica depositada na superfície da cerâmica disponível para se ligar quimicamente ao agente silano ${ }^{19,32}$.
Outra possibilidade de tratamento superficial em cerâmicas policristalinas menos difundido que os anteriores é o condicionamento seletivo por infiltração, que também promove a criação de microrretenções na superfície da zircônia ${ }^{36,37}$.

Além de um adequado padrão de porosidade gerado na superfície da cerâmica, o emprego de um agente de união silano é consolidado na literatura para que se obtenha uma adequada adesão entre cerâmica e substrato ${ }^{2,3,7,13}$. Esses agentes de união à base de silano apresentam uma característica bifuncional, permitindo interagir com o dióxido de silício presente na superfície cerâmica e com a matriz orgânica do adesivo resinoso. Seja no tratamento de superfície de cerâmicas ácido-sensíveis, seja no tratamento de cerâmicas parcialmente cristalinas ou policristalinas, a silanização é ainda considerada uma etapa indispensável na obtenção de uma união bem-sucedida ${ }^{13,19,26,27,29,31,32,35-37}$.

Com base no princípio de promover irregularidades na superfície da cerâmica para promover a adesão aos cimentos resinosos, foi proposto o emprego de lasers como tratamento de superfície ${ }^{38-40}$, que atuariam removendo partículas da superfície por meio de microexplosões e ablasão por vaporização. Porém, ainda que alguns autores tenham obtido resultados positivos nos valores de resistência de união entre zircônia e o cimento resinoso com o emprego de laser de Er:YAG ${ }^{40}$ (érbio: ítrio - alumínio - granada) e de laser de $\mathrm{CO}_{2}{ }^{38}$ (dióxido de carbono), mais estudos são necessários para verificar o efeito das mudanças de temperatura geradas pelo laser nas cerâmicas.

Outra maneira de otimizar a união às cerâmicas policristalinas está relacionada ao uso de adesivos resinosos contendo o monômero MDP, que mostrou bons resultados na resistência de união à zircônia. Essa abordagem não deve ser encarada como uma maneira de substituir os tratamentos superficiais nas restaurações cerâmicas, mas como um complemento para alcançar uma adesão mais efetiva à zircônia $^{23,41-43}$

\section{Conclusão}

Vitrocerâmicas são adequadamente tratadas por meio de condicionamento com HF, em concentração e tempo correspondentes ao seu conteúdo cristalino, seguido de silanização. Cerâmicas ácido-resistentes tratadas com métodos como condicionamento seletivo por infiltração ou revestimento triboquímico com sílica, seguidos de silanização e utilização de primers ou cimentos resinosos contendo monômero MDP, resultam em maiores valores de resistência de união. 


\section{Abstract}

Objective: this study presents a review of the literature on the types of surface treatment available for bonding cement to ceramic systems. Literature review: although there is some consensus on the adhesive bonding to glass-ceramics, there is still no unanimity regarding the adhesion of high-resistance polycrystalline ceramics. Based on the literature available, the preferential treatment for glass ceramics is still hydrofluoric acid (HF) etching, at concentration and time corresponding to its crystalline content, followed by silanization. For polycrystalline ceramics, methods such as selective conditioning by infiltration or tribochemical coating with silica followed by silanization and the use of primers or resin cement containing MDP monomer resulted in high bond strength values. Final considerations: glass ceramics are suitably treated by HF etching followed by silanization. Higher bond strength values for polycrystalline ceramics were obtained after treatment with selective conditioning by infiltration or silicatization followed by silanization, in addition to the use of primers or resin cement containing MDP monomer. However, additional in vitro studies are needed to define the best treatment option for such materials.

Keywords: Ceramics. Cementation. Permanent dental restoration.

\section{Referências}

1. Rashid H, Sheikh Z, Misbahuddin S, Kazmi MR, Qureshi S, Uddin MZ. Advancements in all-ceramics for dental restorations and their effect on the wear of opposing dentition. Eur J Dent 2016; 10(4):583-8.

2. Cotes C, de Carvalho RF, Kimpara ET, Leite FP, Ozcan M. Can heat treatment procedures of pre-hydrolyzed silane replace hydrofluoric acid in the adhesion of resin cement to feldspathic ceramic? J Adhes Dent 2013; 15(6):569-74.

3. Corazza PH, Cavalcanti SC, Queiroz JR, Bottino MA, Valandro LF. Effect of post-silanization heat treatments of silanized feldspathic ceramic on adhesion to resin cement. J Adhes Dent 2013; 15(5):473-9.

4. Peumans M, Valjakova EB, De Munck J, Mishevska CB, Van Meerbeek B. Bonding effectiveness of luting composites to different CAD/CAM materials. J Adhes Dent 2016; 18(4):289-302.

5. Santos MJMC, Costa MD, Rubo JH, Pegoraro LF, Santos GCJ. Current all-ceramic systems in dentistry: a review. Compend Contin Educ Dent 2015; 36(1):31-7, 40.

6. Kelly JR, Benetti P. Ceramic materials in dentistry: historical evolution and current practice. Aust Dent J 2011; 56(Suppl 1):84-96.

7. Fabianelli A, Pollington S, Papacchini F, Goracci C, Cantoro A, Ferrari M, et al. The effect of different surface treatments on bond strength between leucite reinforced feldspathic ceramic and composite resin. J Dent 2010; 38(1):39-43.

8. Della Bona A, Anusavice KJ. Microstructure, composition, and etching topography of dental ceramics. Int J Prosthodont 2002; 15(2):159-67.

9. el-Mowafy $\mathrm{O}$. The use of resin cements in restorative dentistry to overcome retention problems. J Can Dent Assoc 2001; 67(2):97-102.
10. Blatz MB, Sadan A, Kern M. Resin-ceramic bonding: a review of the literature. J Prosthet Dent 2003; 89(3):268-74.

11. Akyil MS, Yilmaz A, Bayindir F, Duymus ZY. Microtensile bond strength of resin cement to a feldspathic ceramic. Photomed Laser Surg 2011; 29(3):197-203.

12. de Oyague RC, Monticelli F, Toledano M, Osorio E, Ferrari $\mathrm{M}$, Osorio R. Influence of surface treatments and resin cement selection on bonding to densely-sintered zirconium-oxide ceramic. Dent Mater 2009; 25(2):172-9.

13. Brentel AS, Ozcan M, Valandro LF, Alarca LG, Amaral R, Bottino MA. Microtensile bond strength of a resin cement to feldpathic ceramic after different etching and silanization regimens in dry and aged conditions. Dent Mater 2007; 23(11):1323-31.

14. Shiu P, De Souza-Zaroni WC, Eduardo C de P, Youssef MN. Effect of feldspathic ceramic surface treatments on bond strength to resin cement. Photomed Laser Surg 2007; 25(4):291-6.

15. Yavuz T, Eraslan O. The effect of silane applied to glass ceramics on surface structure and bonding strength at different temperatures. J Adv Prosthodont 2016; 8(2):75-84.

16. Kiyan VH, Saraceni CHC, da Silveira BL, Aranha ACC, Eduardo $\mathrm{C}$ da P. The influence of internal surface treatments on tensile bond strength for two ceramic systems. Oper Dent 2007; 32(5):457-65.

17. Senyilmaz DP, Palin WM, Shortall ACC, Burke FJT. The effect of surface preparation and luting agent on bond strength to a zirconium-based ceramic. Oper Dent 2007; 32(6):623-30

18. Hallmann L, Ulmer P, Lehmann F, Wille S, Polonskyi O, Johannes M, et al. Effect of surface modifications on the bond strength of zirconia ceramic with resin cement resin. Dent Mater 2016; 32(5):631-9.

19. Wandscher VF, Prochnow C, Rippe MP, Dorneles LS, Callegari GL, Baldissara P, et al. Retentive strength of Y-TZP crowns: comparison of different silica coating methods on the intaglio surfaces. Oper Dent 2017; 42(5):E121-33.

20. Spohr AM, Borges GA, Junior LHB, Mota EG, Oshima HMS. Surface modification of In-Ceram Zirconia ceramic by Nd:YAG laser, Rocatec system, or aluminum oxide sandblasting and its bond strength to a resin cement. Photomed Laser Surg 2008; 26(3):203-8.

21. Kasraei S, Rezaei-Soufi L, Yarmohamadi E, Shabani A. Effect of $\mathrm{CO} 2$ and Nd:YAG lasers on shear bond strength of resin cement to zirconia ceramic. J Dent (Tehran) 2015; 12(9):686-94.

22. Ahrari F, Boruziniat A, Alirezaei M. Surface treatment with a fractional $\mathrm{CO} 2$ laser enhances shear bond strength of resin cement to zirconia. Laser Ther 2016; 25(1):19-26.

23. Tanis MC, Akcaboy C. Effects of different surface treatment methods and MDP monomer on resin cementation of zirconia ceramics an in vitro study. J Lasers Med Sci 2015; 6(4):174-81.

24. Arai M, Takagaki T, Takahashi A, Tagami J. The role of functional phosphoric acid ester monomers in the surface treatment of yttria-stabilized tetragonal zirconia polycrystals. Dent Mater J 2017; 36(2):190-4.

25. Venturini AB, Prochnow C, Rambo D, Gundel A, Valandro LF. Effect of hydrofluoric acid concentration on resin adhesion to a feldspathic ceramic. J Adhes Dent 2015; 17(4):313-20.

26. Neis CA, Albuquerque NLG, Albuquerque I de S, Gomes EA, Souza-Filho CB de, Feitosa VP, et al. Surface treatments for repair of feldspathic, leucite - and lithium disilicate-reinforced glass ceramics using composite resin. Braz Dent J 2015; 26(2):152-5. 
27. Stella JPF, Oliveira AB, Nojima LI, Marquezan M. Four chemical methods of porcelain conditioning and their influence over bond strength and surface integrity. Dental Press J Orthod 2015; 20(4):51-6.

28. Della Bona A, Mecholsky JJJ, Barrett AA, Griggs JA. Characterization of glass-infiltrated alumina-based ceramics. Dent Mater 2008; 24(11):1568-74.

29. Panah FG, Rezai SMM, Ahmadian L. The influence of ceramic surface treatments on the micro-shear bond strength of composite resin to IPS Empress 2. J Prosthodont 2008; 17(5):409-14.

30. Vichi A, Carrabba M, Paravina R, Ferrari M. Translucency of ceramic materials for CEREC CAD/CAM system. J Esthet Restor Dent 2014; 26(4):224-31.

31. Venturini AB, Prochnow C, May LG, Bottino MC, Felipe Valandro L. Influence of hydrofluoric acid concentration on the flexural strength of a feldspathic ceramic. J Mech Behav Biomed Mater 2015; 48:241-8.

32. Eduardo C de P, Bello-Silva MS, Moretto SG, Cesar PF, de Freitas PM. Microtensile bond strength of composite resin to glass-infiltrated alumina composite conditioned with Er,Cr:YSGG laser. Lasers Med Sci 2012; 27(1):7-14.

33. Pattanaik S, Wadkar AP. Effect of etchant variability on shear bond strength of all ceramic restorations - an in vitro study. J Indian Prosthodont Soc 2011; 11(1):55-62.

34. Aboushelib MN, Sleem D. Microtensile bond strength of lithium disilicate ceramics to resin adhesives. J Adhes Dent 2014; 16(6):547-52.

35. Oyague RC, Osorio R, da Silveira BL, Toledano M. Comparison of bond stability between dual-cure resin cements and pretreated glass-infiltrated alumina ceramics. Photomed Laser Surg 2011; 29(7):465-75.

36. Roman-Rodriguez JL, Roig-Vanaclocha A, Fons-Font A, Granell-Ruiz M, Sola-Ruiz MF, Amigo-Borras V, et al. In vitro experimental study of bonding between aluminium oxide ceramics and resin cements. Med Oral Patol Oral Cir Bucal 2010; 15(1):e95-100.

37. Aboushelib MN, Kleverlaan CJ, Feilzer AJ. Selective infiltration-etching technique for a strong and durable bond of resin cements to zirconia-based materials. J Prosthet Dent 2007; 98(5):379-88.

38. Dede DO, Yenisey M, Rona N, Ongoz Dede F. Effects of laser treatment on the bond strength of differently sintered zirconia ceramics. Photomed Laser Surg 2016; 34(7):276-83.

39. Ural C, Kulunk T, Kulunk S, Kurt M. The effect of laser treatment on bonding between zirconia ceramic surface and resin cement. Acta Odontol Scand 2010; 68(6):354-9.

40. Akin H, Tugut F, Akin GE, Guney U, Mutaf B. Effect of Er:YAG laser application on the shear bond strength and microleakage between resin cements and Y-TZP ceramics. Lasers Med Sci 2012; 27(2):333-8.

41. Akhavan Zanjani V, Ahmadi H, Nateghifard A, Ghasemi A, Torabzadeh H, Abdoh Tabrizi M, et al. Effect of different laser surface treatment on microshear bond strength between zirconia ceramic and resin cement. J Investig Clin Dent $2015 ; 6(4): 294-300$.

42. Wolfart M, Lehmann F, Wolfart S, Kern M. Durability of the resin bond strength to zirconia ceramic after using different surface conditioning methods. Dent Mater 2007; 23(1):45-50.

43. Shin Y-J, Shin Y, Yi Y-A, Kim J, Lee I-B, Cho B-H, et al. Evaluation of the shear bond strength of resin cement to Y-TZP ceramic after different surface treatments. Scanning 2014; 36(5):479-86.
Endereço para correspondência:

Verônica Pereira de Lima

Rua Gonçalves Chaves, 457, Centro

96015-560, Pelotas, Rio Grande do Sul, Brasil

Telefone: (53) 3222-4439

E-mail: veronica.vpl@gmail.com

Recebido: 30/01/18. Aceito: 26/03/18 